\title{
Novel anti-SARS-CoV-2 mechanisms of fusion broad range anti- infective protein ricin A chain mutant-pokeweed antiviral protein 1 (RTAM-PAP1) in silico
}

\author{
Yasser Hassan ( $\nabla$ yhassa@gmail.com ) \\ Ophiuchus Medicine Inc. https://orcid.org/0000-0001-6828-3092 \\ Sherry Ogg \\ Johns Hopkins University \\ Hui Ge \\ AscentGene Inc.
}

\section{Short Report}

Keywords: fusion proteins; ricin; pokeweed antiviral protein, COVID-19; SARS-CoV-2, antiviral agent, ribosome-inactivating proteins

Posted Date: June 23rd, 2020

DOI: https://doi.org/10.21203/rs.3.rs-32952/v2

License: (c) (1) This work is licensed under a Creative Commons Attribution 4.0 International License. Read Full License

Version of Record: A version of this preprint was published on September 17th, 2020. See the published version at https://doi.org/10.3390/toxins12090602. 


\section{Abstract}

A deadly pandemic named COVID-19 caused by a new coronavirus SARS- CoV-2 has emerged in 2019 and is still spreading globally at a dangerous pace. As of today, there are no proven vaccines, therapies or even strategies to fight off this virus. Here, we describe the in silico results of a novel broad range antiinfective fusion protein RTAM-PAP1 against the various key pro- teins of SARS-CoV-2 using the latest protein-ligand docking software. RTAM-PAP1 was compared against the SARS-CoV-2 B38 antibody, ricin A chain, pokeweed antiviral protein from leaves and the lectin griffithsin using CoDockPP special COVID19 version. These experiments revealed novel binding mechanisms of RTAM-PAP1 with high affinity to numerous targets with anti-SARS-CoV-2 effects. RTAM-PAP1 was further characterized in a preliminary toxicity study in mice and was found to likely be a potent anti-SARS-CoV-2 agent. These findings might lead to the discovery of novel SARS-CoV-2 targets and therapeutic protein structures.

\section{Brief Report}

A new global pandemic disease named COVID-19 has emerged and is still spreading at alarming rates at the time of this report. COVID-19 can cause severe symptoms such as damaging inflammatory response, fever or severe respiratory illness and lead to death. The causative agent of COVID-19 was found to be a novel coronavirus closely related to the severe acute respiratory syndrome coronavirus (SARS-CoV) based on the latest phylogenetic analysis ${ }^{1-3}$. There are nonetheless some major essential differences in their genetic make-up that led to their very different behaviors. Indeed, SARS-CoV-2, as it is called now, appears to have a high transmissibility from person to person and antibodies that could inhibit SARS-CoV are not functional on SARS-CoV-2 $2,4-5$. Despite global efforts, we still lack an effective antiviral strategy, drug or vaccine to fight off this virus with the growing fear that SARS-COV-2 may become another endemic virus in our communities.

In order to lower the costs of and speed up the drug discovery phase, numerous researchers have utilized in silico tools such as protein-ligand docking software to screen for traditional compounds that could bind to and inhibit the key proteins present in SARS-CoV-2, highlighting their potential antiviral activity ${ }^{6}$. The major targets for these compounds include SARS-CoV-2 key proteins 3-chymotrypsin-like protease (Mpro), papain like protease (PLpro), RNA-dependent RNA polymerase (RdRp), small envelope protein (E), membrane protein $(M)$ and spike $(S)$ proteins. The S proteins interact directly with human angiotensinconverting enzyme (ACE2), allowing the virus to enter the cells. The $S$ protein is a class I fusion protein consisting of S1 and S2 domains with the receptor binding domain (RBD) located on the S1 domain ${ }^{4}$. The RBD is the main target of antibodies and fusion inhibitors in development such as the human convalescent COVID-19 patient-origin B38 antibody (B38) and plant lectin griffithsin (GRFT).

Here, we report the in silico potent antiviral activity against SARS-CoV-2 of a previously discussed novel broad spectrum anti-infective fusion protein between a mutant of the ricin A chain and pokeweed antiviral protein isolated from leaves (RTAM-PAP1) from Ricinus communis and Phytolacca americana respectively ${ }^{7}$. RTAM-PAP1 activity was compared with that of the B38, ricin A chain (RTA), pokeweed 
antiviral protein isolated from leaves (PAP1) and GRFT. Their binding and inhibiting capacities were evaluated against the major key proteins of SARS-CoV-2 using the latest peptide-ligand docking software $e^{8-13}$.

The 3D structure of RTAM-PAP1 was obtained as previously described ${ }^{7}$ and those of RTA, PAP1, B38, and GRFT were retrieved in protein data bank (PDB) format from the Research Collaboratory for Structural Bioinformatics (RCSB) website (https://www.rcsb.org/). A knowledge-based scoring docking prediction was done for all the compounds against S, S1 RBD and M using CoDockPP global docking. An additional run was done for ACE2 and human SARS-CoV antibody CR3022 against S1 RBD as reference ${ }^{5}$. The 3D structures of all the key proteins and ACE2 were already available at the software site in this "covid-19 targets docking only" version. The peptide/antibody-ligand version was used as small molecules docking software is not suited for these types of compounds. The generated 3D model of B38, ACE2 and CR3022 bound to S1 RBD were comparable to available crystallography of the same complexes in RCSB (access: 7BZ5, 6M0J and 6W41 respectively) with some deviations (results not shown). However, the greater binding affinity and fusion inhibiting activity of B38 compared to CR3022 for S1 RBD was observed in accordance with published in vitro results ${ }^{1,3,5}$. Indeed, B38 was found to have a dissociation constant of $70.1 \mathrm{nM}$ with a complete inhibition of ACE2 binding to S1 RBD compared to CR3022's dissociation constant of $115 \mathrm{nM}$ with no inhibition of ACE2 binding. The difference in inhibition of ACE2 binding to S1 RBD is due to their binding conformation to S1 RBD. However, ACE2 binding to S1 RBD was found to have the smallest dissociation constant in literature with a value ranging from 4 to $15 \mathrm{nM}$. The results for the first and last model (out of the top ten generated) of each compounds in complex with S, S1 RBD and $\mathrm{M}$ are presented in Table 1.a. B38 has the highest overall binding affinity of the lot with a binding energy ranging from -449 to $-300 \mathrm{kcal} / \mathrm{mol}$, as expected. ACE2's binding energy was between -314 to -246 $\mathrm{kcal} / \mathrm{mol}$ for S1 RBD. RTAM-PAP1 is very comparable to B38, with an overall higher binding affinity (lower binding energy) than all of the other compounds tested against the S, S1 RBD and M key proteins, sometimes higher than that of B38 with $-469 \mathrm{kcal} / \mathrm{mol}$ for $\mathrm{M}$, for example. The high binding affinity of RTAM-PAP1 and B38 to S, S1 and M may be explained by the fact that the $M$ epitope is very similar in structure to S1 RBD (figure 1.a) 1,3. RTA binding affinity is similar to RTAM-PAP1 to a certain extent and GRFT and PAP1 are very comparable. The same higher binding affinity behavior for RTAM-PAP1 was observed with Mpro, PLpro, E and RdRp when compared to PAP1, GRFT and RTA (Table 1.b). All of the tested compounds showed potentially inhibiting biding conformations to the various key proteins based on the 3D structures of the complexes formed (results not shown). These results indicate that the fusion between RTAM and PAP1 allowed RTAM-PAP1 to be more stable across the different possible binding conformations with a higher binding affinity than either of its moieties alone when in complex with SARSCoV-2 key proteins.

B38 was found to have an inhibition of the cytopathic effect of 50\% (EC50) against SARS-CoV-2 simultaneous infection in Vero Cells in vitro at the concentration of $0.177 \mathrm{ug} / \mathrm{ml}$. It was further demonstrated that B38 was effective in mice post-infection ${ }^{1}$. GRFT was found to have low pre-infection EC50 on different strains of SARS-CoV in cytoprotection (CPE) assays in vitro $(0.6-1.2 \mathrm{ug} / \mathrm{ml})$ and 
effective in mice pre-infection ${ }^{14}$. RTA was shown in literature to have high binding affinity to many viral proteins ${ }^{15-16}$. PAP1 has a broad range antiviral activity against numerous infections both in vitro and in clinical trials ${ }^{17-18}$. An earlier different version of RTAM-PAP1 was shown to have potent broad range antiviral activity at low post-infection EC50 $(0.002-12.3 \mathrm{ug} / \mathrm{ml})$ against human immunodeficiency virus-I (HIV), hepatitis B virus (HBV), hepatitis C virus (HCV), Zika virus (Zika) and human coronavirus 229E (HCoV229E) in CPE assays in vitro ${ }^{7,19}$. RTA and PAP1 have been observed to have a drastic increase in viral inhibition activity if administered pre-infection both in vitro and in vivo at sub-toxic dosages ${ }^{20-23}$ with potent antiviral mechanisms, from viral DNA/RNA depurination, viral proteins synthesis inhibition, viral cell entry inhibition to apoptosis induction of infected cells via a preferential virus infected cell entry mechanism ${ }^{7}$.

Nonetheless, such high affinity of RTAM-PAP1 to many key proteins of SARS-CoV-2 is uncommon. Yet, the most surprising part of the generated models was the discovery of unique binding mechanisms of RTAM-PAP1 with potential inhibiting activity by hindering viral entry and cellular machinery. This discovery might explain the previously observed gain of function of RTAM-PAP $1^{7}$ by means of the acquired ability to simultaneously bind the target with both moieties with high affinity, i.e. increasing the docking sites from 86 to 102 for single moiety binding and simultaneous binding to S1 RBD respectively, for example.

In order to confirm these findings, RTAM-PAP1 was run against SARS-CoV-2 S1 and M using different docking programs (Zdock and HADDOCK2.2) with the known active residues in RCSB. The synergetic binding of RTAM-PAP1 was confirmed and the generated models for $\mathrm{M}$ are shown in figure 1.b-d. Although the model generated by HADDOCK2.2 returned a more important role for PAP1 than RTAM, the simultaneous binding of both moieties can clearly be seen when in complex with $M$ with an increase in docking sites from 62 for single moiety binding to 96 for simultaneous binding of both moieties (Zdock model). This might significantly increase RTAM-PAP1 anti-SARS-CoV-2 activity. It can be concluded from these results and those previously acquired in vitro that the fusion of RTAM and PAP1 via the flexible linker conferred greater structure stability, enhanced activities, new binding sites and mechanisms and also, potentially, novel functions to RTAM-PAP1.

For those reasons, a short toxicity study of RTAM-PAP1 was conducted in BALB/c mice to determine the potential maximum tolerated dose. Adverse clinical signs were observed at a single bolus intravenous administration of $3 \mathrm{mg} / \mathrm{kg}$ of RTAM-PAP1 with up-regulation of IP-10, KC and MCP-10 chemokines from 14 cytokines/chemokines assessed (results not shown). These results are in line with previously described homopolymers of ribosome inactivating proteins and confirm an in vivo behavior intermediate between that of native ribosome inactivating proteins and of immunotoxins ${ }^{24-25}$.

In conclusion, given the very high affinity for SARS-CoV-2 key proteins, the previous antiviral results in vitro, the newly discovered mechanisms, the preliminary in vivo profile, potent bioactivities across the assays and preferential entry into virus infected cells as opposed to non-infected cells, it is the opinion of the authors that this novel $\mathrm{N}$-glycosidase ribosome inactivating fusion protein be tested against SARS- 
CoV-2 in vitro and in vivo. It would be the first tested therapeutic utilizing this particular strategy against COVID-19 and might make a difference at subtoxic dosages and open the doors for the discovery of novel SARS-CoV-2 targets and therapeutic protein structures.

Table 1.a

\begin{tabular}{|c|c|c|c|c|c|c|}
\hline & \multicolumn{2}{|c|}{ S1 RBD } & \multicolumn{2}{c|}{ Spike Trimer } & \multicolumn{2}{c|}{ Membrane Protein } \\
\hline & top 1 & top 10 & top 1 & top 10 & top 1 & top 10 \\
\hline ACE2 & -314 & -246 & & & & \\
\hline Compounds & & & & & & \\
\hline CR3022 & -347 & -285 & & & & \\
\hline B38 Anti- & -367 & -300 & -385 & -297 & -449 & -359 \\
\hline body & -273 & -239 & -283 & -250 & -280 & -265 \\
\hline GRFT & -322 & -282 & -325 & $\mathbf{- 2 9 8}$ & -469 & $-\mathbf{3 9 3}$ \\
\hline RTAM-PAP1 & -322 & -278 & -313 & -275 & -387 & -348 \\
\hline RTA & -329 & -233 & -281 & -255 & -300 & -266 \\
\hline PAP1 & -269 & & & & & \\
\hline
\end{tabular}

This table represents the binding energies in $\mathrm{kcal} / \mathrm{mol}$ for the models generated by CoDockPP for each compounds in complex with the outer virus envelope proteins. The top 1 is the model with the lowest binding energy (highest binding affinity) and the top 10 is the $10^{\text {th }}$ model with the lowest binding energy. The lowest energy for the top1 and top10 models for each complex is in bold. 


\begin{tabular}{|c|c|c|c|c|c|c|c|c|}
\hline \multirow[t]{2}{*}{$\begin{array}{c}\text { Key Pro- } \\
\text { teins }\end{array}$} & \multicolumn{2}{|c|}{ Mpro } & \multicolumn{2}{|c|}{ Plpro } & \multicolumn{2}{|c|}{ RdRp } & \multicolumn{2}{|c|}{ E Protein } \\
\hline & top 1 & top 10 & top 1 & $\begin{array}{c}\text { top } \\
10\end{array}$ & top 1 & $\begin{array}{c}\text { top } \\
10\end{array}$ & top 1 & top 10 \\
\hline $\begin{array}{l}\text { Com- } \\
\text { pounds }\end{array}$ & & & & & & & & \\
\hline GRFT & -228 & -198 & -234 & -209 & -267 & -248 & -258 & -242 \\
\hline $\begin{array}{c}\text { RTAM- } \\
\text { PAP1 }\end{array}$ & -301 & -266 & -276 & -259 & -332 & -301 & -363 & -306 \\
\hline RTA & -299 & -260 & -283 & -254 & -304 & -277 & -314 & -281 \\
\hline PAP1 & -246 & -207 & -225 & -188 & -244 & -228 & -244 & -229 \\
\hline
\end{tabular}

This table represents the binding energies in $\mathrm{kcal} / \mathrm{mol}$ for the models generated by CoDockPP for each compound less the antibody with the viral proteins important for cellular machinery. The top 1 is the model with the lowest binding energy (highest binding affinity) and the top10 is the $10^{\text {th }}$ model with the lowest binding energy. The lowest energy for the top 1 and top10 models for each complex is in bold.

\section{Materials And Methods 2.1. Protein Modeling}

\subsubsection{Generation of 3D structures}

The molecular 3D structure of RTAM-PAP1 was already available from previous work ${ }^{7}$ and is available in the supplementary files. The 3D models for RTA, PAP1, B38, CR3022 and GRFT were retrieved from RCSB (https://www.rcsb.org), in pdb format with the following PDB ID: 4MX5, 1PAG, 7BZ5, 6W41 and 3LL2 respectively). The 3D models for S, Mpro, PLpro, ACE2, RdRp, E and M were retrieved directly from the 
CoDockPP site (https://ncov.schanglab.org.cn/). The 3D models for the B38-S1 and ACE2-S1 complexes were also retrieved from RCSB, 7BZ5 and 6LZG respectively, for comparison with CoDockPP outputs.

\subsubsection{Structure modeling}

The structure of the bound complexes were generated by CoDockPP using the ambiguous peptide-ligand computations $^{8-10}$. The B38-S1, CR3022-S1 and ACE2-S1 were compared by superimposition on the available crystallography in RCSB using MATRAS Pairwise 3D alignment (http://strcomp.protein.osakau.ac.jp/matras/matras_pair.html). Additional models of the RTAM-PAP1-S1 and RTAM-PAP1-M complexes were generated using ZDOCK and HADDOCK2. $2^{11-13}$ with the RCSB available active residues as inputs for each protein. The putative active residues for RTAM-PAP1 were previously generated ${ }^{7}$ and are available in the supplementary files. All models were viewed using Jmol.

\subsection{E. coli in vivo expression system and Rabbit Reticulate Lysate protein synthesis inhibition}

\subsubsection{Protein expression and purification}

RTAM-PAP1 was produced and purified as previously described ${ }^{7}$. In brief, the vector pET30a-6H-RPAP1 was generated and validated by DNA sequencing before being transformed into $E$. coli BL21(DE3) cells (NEB). Expression of the proteins were examined from individual clones and analyzed by either Western blot using a monoclonal antibody specific to ricin A chain (ThermoFisher, RA999) or SDS gel stained with Comassie blue (ThermoFisher). Optimal conditions were determined and protein production induced in the presence of $1 \mathrm{mM}$ IPTG from $1 \mathrm{~L}$ culture. The bacteria were then harvested by centrifugation, followed by lysing the cell pellets with $50 \mathrm{ml}$ of lysis buffer $(50 \mathrm{mM}$ Tris-Cl, $150 \mathrm{mM} \mathrm{NaCl}, 0.2 \%$ Triton $\mathrm{X} 100$ and $0.5 \mathrm{mM}$ EDTA). After sonication $(3 \times 2 \mathrm{~min})$, the soluble lysates were recovered by centrifugation at $35 \mathrm{~K} \mathrm{rpm}$ for $40 \mathrm{~min}$. The soluble proteins were then purified by the combination of affinity and conventional chromatographic methods from soluble lysates (please contact the authors for more details). The purification of the native RTAM-PAP1 from soluble lysate was achieved by affinity versus His-tag on Nisepharose column (GE Healthcare). After extensive washed with the lysis buffer, loosely bound proteins were eluted with the lysis buffer containing $40 \mathrm{mM}$ Imidazole (140). RTAM-PAP1 proteins were eluted with the elution buffer (20mM Tris-Cl, pH7.9, $100 \mathrm{mM} \mathrm{NaCl}, 1 \mathrm{mM}$ EDTA and $300 \mathrm{mM}$ Imidazole). A second purification step using Hydroxylapatite column (GE Healthcare) was used to further separate RTAM-PAP1 from co-purified host proteins. A third purification step, gel filtration on a fast protein liquid chromatography (FPLC) column of Superose 12 (GE Healthcare), was necessary to completely get rid of degraded or/and premature protein products. The resulting mixture went through the endotoxin removal process by using a proprietary technology developed by AscentGene until endotoxin level was less than 
$10 \mathrm{EU} / \mathrm{ml}$. The final product was formulated in the buffer containing 20mM HEPES-Na, pH7.9, 200mM $\mathrm{NaCl}, 0.2 \mathrm{mM} \mathrm{CaCl} 2$ and $0.5 \mathrm{mM}$ EDTA.

\subsubsection{Rabbit Reticulate Lysate protein synthesis inhibition}

The inhibitory activities of RTAM-PAP1 was tested by using the Rabbit Reticulate Lysate TnT® Quick Coupled Transcription/Translation System and the Luciferase Assay System (Promega). Briefly, each transcription/translation reaction was performed according to the instructions for use (IFU) in the presence of a T7 Luciferase reporter DNA, and the Luciferase expression level was determined with a Wallac Microplate Reader. Transcription/translation runs were at three different concentrations to confirm bioactivity.

\subsection{Preliminary toxicity study on mice}

\subsubsection{BALB/c mice}

Female BALB/c mice, aged 6-8 weeks (Charles River Laboratories, Saint-Constant, Quebec, Canada), were used in this study. Female mice were housed in groups of five in individually ventilated cages. Mice were maintained at the National Research Council Canada (NRC) in accordance with the guidelines of the Canadian Council on Animal Care. All procedures performed on animals in this study were in accordance with regulations and guidelines reviewed and approved by the NRC Human Health Therapeutics Ottawa Animal Care Committee.

\subsubsection{Animal Procedures}

RTAM-PAP1 was administered by intravenous (IV) bolus injection of $0.25 \mathrm{ml}$ into the tail vein in a dose escalating manner $(0.03,0.1,0.3,1$ and $3 \mathrm{mg} / \mathrm{kg})$. Control mice received an equivalent volume of vehicle. Dosing was staggered to allow for an initial assessment of tolerability at a particular dose level prior to escalating to a higher dose level. Mice were weighted and evaluated daily for clinical signs for 8 days following administration of RTAM-PAP1. Data were analyzed using GraphPad Prism (GraphPad Software, Inc., San Diego, CA, USA). Statistical significance of the difference between groups was calculated by 1factor or 2-factor ANOVA followed by post-hoc analysis. Differences were considered to be not significant with $p>0.05$.

\section{Declarations}

Ethics approval and consent to participate: Not applicable Consent for publication: Not applicable 
Availability of Data and Materials: "The datasets used and/or analyzed during the current study which are not included are available from the corresponding author on reasonable request"

Conflicts of Interest: "The authors are either directly or indirectly affiliated to Ophiuchus Medicine. Y. H. and S. O. are officers and shareholders of Ophiuchus Medicine. H. G.'s AscentGene is a subcontractor of Ophiuchus Medicine."

Funding: "This work was funded by Ophiuchus Medicine which owns the rights to the patent pending of the fusion protein described in this study. The entire study was done at the request of, by and for Ophiuchus Medicine."

Authors' contributions: $\mathrm{Y}$. H. was responsible for the design, data generation and for analyses of the in silico experiments. Y. H. was a major contributor for writing the manuscript. S. H. reviewed the in silico experiments and helped in the analysis of the data generated. S. H. revised the entire manuscript critically. H. G. was responsible for the protein production, purification and protein inhibition assays. H. G. was responsible for drafting some of the content of the manuscript and revising critically the entire manuscript. National Research Council of Canada was solely responsible for the preliminary toxicity study on mice.

Acknowledgements: "The FP7 WeNMR (project\# 261572), H2020 West-Life (project\# 675858) and the EOSC-hub (project\# 777536) European e-Infrastructure projects are acknowledged for the use of their web portals, which make use of the EGI infrastructure with the dedicated support of CESNET-MCC, INFNPADOVA, NCG-INGRID-PT, TW-NCHC, SURFsara and NIKHEF, and the additional support of the national GRID Initiatives of Belgium, France, Italy, Germany, the Netherlands, Poland, Portugal, Spain, UK, Taiwan and the US Open Science Grid."

\section{References}

1. Wu Y. et al. A noncompeting pair of human neutralizing antibodies block COVID-19 virus binding to its receptor ACE2. Science. 2020 May 13. pii: eabc2241. doi: 10.1126/science.abc2241.

2. Othman $\mathrm{H}$. et al. Interaction of the spike protein RBD from SARS-CoV-2 with ACE2: Similarity with SARS-CoV, hot-spot analysis and effect of the receptor polymorphism. Biochem Biophys Res Commun. 2020 May 14. doi: 10.1016/j.bbrc.2020.05.028.

3. Lan J. et al. Structure of the SARS-CoV-2 spike receptor-binding domain bound to the ACE2 receptor. Nature. 2020 May;581(7807):215-220. doi: 10.1038/s41586-020-2180-5. Epub 2020 Mar 30.

4. Amawi H. et al. COVID-19 pandemic: an overview of epidemiology, pathogenesis, diagnostics and potential vaccines and therapeutics. Ther Deliv. 2020 May 12. doi: 10.4155/tde-2020-0035.

5. Yuan M. et al. A highly conserved cryptic epitope in the receptor binding domain of SARS-CoV-2 and SARS-CoV. Version 2. Science. 2020 May 8;368(6491):630-633. doi:10.1126/science.abb7269. Epub 2020 Apr 3. 
6. Mani JS. et al. Natural product-derived phytochemicals as potential agents against coronaviruses: A review. Virus Res. 2020 Apr 30;284:197989. doi: 10.1016/j.virusres.2020.197989.

7. Hassan Y. et al. Expression of novel fusion antiviral proteins ricin a chain-pokeweed antiviral proteins (RTA-PAPs) in Escherichia coli and their inhibition of protein synthesis and of hepatitis $B$ virus in vitro. BMC Biotechnol. 2018 Aug 6;18(1):47. doi: 10.1186/s12896-018-0458-6.

8. Kong R. et al. COVID-19 Docking Server: An interactive server for docking small molecules, peptides and antibodies against potential targets of COVID-19. 2020. arXiv:2003.00163v1 [q-bio.BM].

9. Kong R. et al. CoDockPP: a multistage approach for global and site-specific protein-protein docking. Journal of Chemical Information and Modeling. 2019, 59(8): 3556-3564.

10. Trott O. and Olson A J. AutoDock Vina: improving the speed and accuracy of docking with a new scoring function, efficient optimization and multithreading. Journal of Computational Chemistry. 2010, 31: 455-461.

11. Pierce BG. Et al. ZDOCK server: interactive docking prediction of protein-protein complexes and symmetric multimers. Bioinformatics. 2014 Jun 15;30(12):1771-3. doi:

10.1093/bioinformatics/btu097. Epub 2014 Feb 14.

12. C.P van Zundert et al. "The HADDOCK2.2 webserver: User-friendly integrative modeling of biomolecular complexes.", J. Mol. Biol., 428, 720-725 (2015).

13. Wassenaar T.A. et al. "WeNMR: Structural Biology on the Grid.", J. Grid. Comp., 10, 743-767 (2012).

14. O'Keefe Br. Et al. Broad-spectrum in vitro activity and in vivo efficacy of the antiviral protein griffithsin against emerging viruses of the family Coronaviridae. J Virol. 2010 Mar;84(5):2511-21. doi: 10.1128/JVI.02322-09. Epub 2009 Dec 23. Erratum in: J Virol. 2010 May;84(10):5456.

15. Olson MC. et al. Ribosomal inhibitory proteins from plants inhibit HIV-1 replication in acutely infected peripheral blood mononuclear cells. AIDS Res Hum Retroviruses. 1991 Dec;7(12):1025-30.

16. Ko SM et al. Detection of hepatitis A virus in seeded oyster digestive tissue by ricin A-linked magnetic separation combined with reverse transcription PCR. J Food Prot. 2015 May;78(5):1046-51. doi: 10.4315/0362-028X.JFP-14-540.

17. Domashevskiy AV and Goss DJ. Pokeweed antiviral protein, a ribosome inactivating protein: activity, inhibition and prospects. Toxins (Basel). 2015;7(2):274-98.

18. Uckun FM. Et al. Toxicity, biological activity, and pharmacokinetics of TXU (anti-CD7)-pokeweed antiviral protein in chimpanzees and adult patients infected with human immunodeficiency virus. $J$ Pharmacol Exp Ther. 1999 Dec;291(3):1301-7.

19. Hassan Y. and Ogg S. WO/2019/204902, The World Intellectual Property Organization (WIPO), 2019.

20. Teltow GJ et al. Inhibition of herpes simplex virus DNA synthesis by pokeweed antiviral protein. Antimicrob Agents Chemother. 1983 Mar;23(3):390-6. doi: 10.1128/aac.23.3.390

21. Tomlinson JA. et al. The inhibition of infection by cucumber mosaic virus and influenza virus by extracts from Phytolacca americana. J Gen Virol. 1974;22(2):225-32. 
22. Ussery MA. et al. Inhibition of poliovirus replication by a plant antiviral peptide. Ann N Y Acad Sci. 1977;284:431-40. doi: 10.1111/j.1749-6632.1977.tb21979.x.

23. Ishag HZ. et al. Inhibition of Japanese encephalitis virus infection in vitro and in vivo by pokeweed antiviral protein. Virus Res. 2013;171(1):89-96. doi: 10.1016/j.virusres.2012.10.032.

24. Barbieri L. et al. Blood clearance and organ distribution and tissue concentration of native, homopolymerized and IgG-conjugated ribosome-inactivating proteins. Xenobiotica. 1990 Dec;20(12):1331-41.

25. Battelli MG. et al. Toxicity of, and histological lesions caused by, ribosome-inactivating proteins, their IgG-conjugates, and their homopolymers. APMIS. 1990 Jul;98(7):585-93.

\section{Figures}
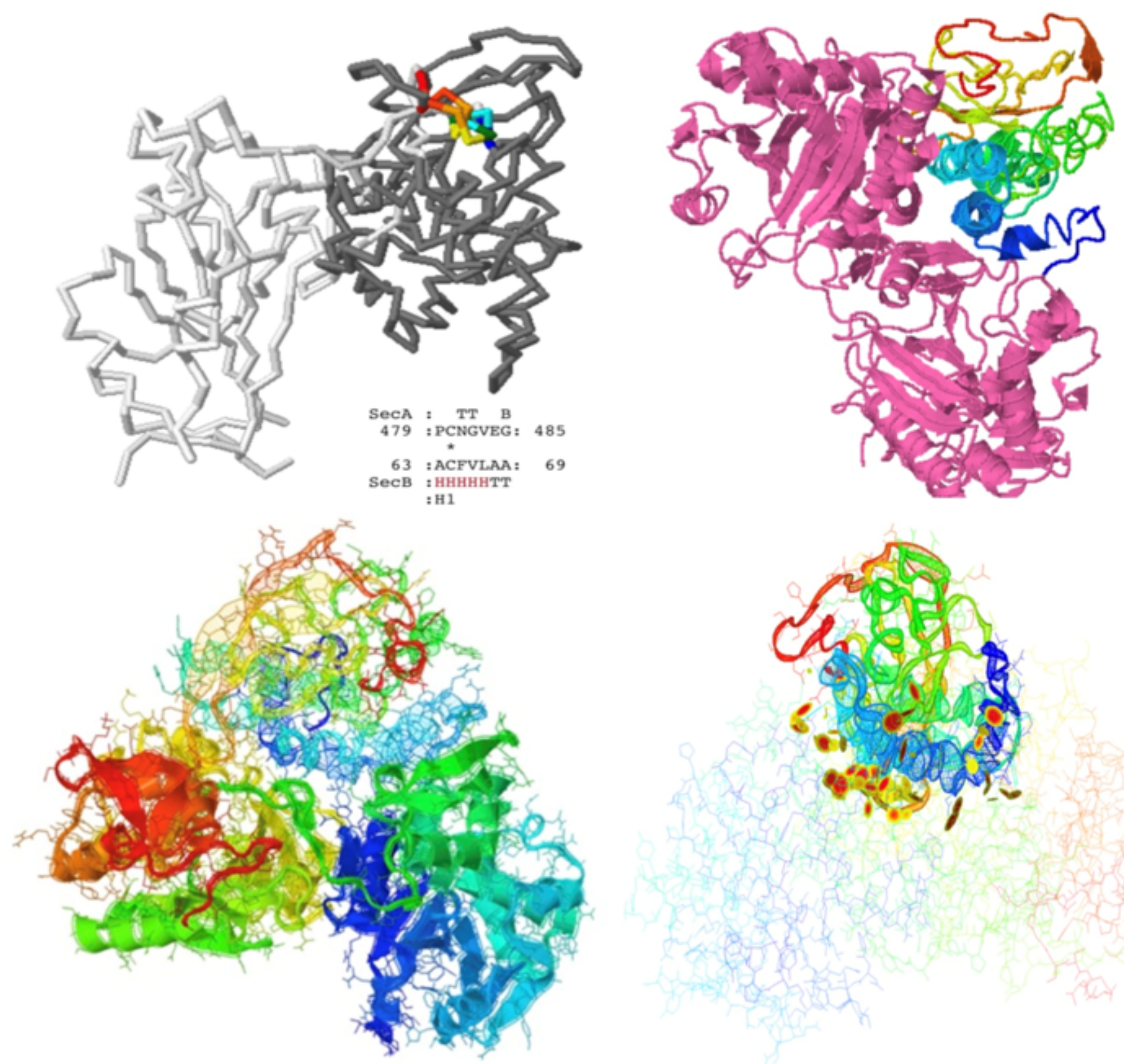
Figure 1

a) 3D structure comparison by MATRAS of $M$ protein epitope to $S 1$ protein. $M$ epitope sequence $(\mathrm{SecB})$ is not similar to S1 RBS, yet the structure is as shown in rainbow colors. b) Top model generated by CoDockPP of RTAM-PAP1 (magenta, RTAM being on the left side) in complex with M protein (up). c) Top model generated by HADDOCL2.2 with a score of -125 . PAP1 is on the left side to denote its more important role than in other models. d) Top model generated by Zdock with RTAM-PAP1 in backbone format and RTAM on the left side in blue. The colored disks depict the binding contact sites. The disks indicate where the van der Waals radii of atoms overlaps and the colors how close the contact is: yellow = close , orange $=$ touching, and red $=$ overlapping. For $b-\mathrm{d}$, the rainbow colors (blue to red) depict the $\mathrm{N}$ terminal to $\mathrm{C}$ terminal of the colored protein (models viewed using Jmol).

\section{Supplementary Files}

This is a list of supplementary files associated with this preprint. Click to download.

- RTAMPAP1activesitefromlTasser.png

- RTAMdvadiPAP1.pdb 